\title{
Reconstrucción del estado de las fortificaciones de Fuentarrabía a principios del siglo XVII a través de un plano atribuido a Matteo Neroni
}

\author{
Víctor Echarri Iribarren ${ }^{a}$, Roberto Tomás Yáñez Pacios ${ }^{b}$ \\ Universidad de Alicante, Alicante, Spain, ㄱictor.Echarri@ua.es, brtyp@alu.ua.es
}

\begin{abstract}
Fuenterrabía, one of the most outstanding strongholds of the Basque Country, historically, has always been a strategic checkpoint in the Spanish-French border. Its bastioned fortification system was, from the sixteenth century, of fundamental importance in the development of armed conflicts between these two nations. It was one of the first Spanish strongholds to adopt the initial Renaissancist design of the bastion. Military engineers continuously developed fortification projects to adequately withstand the advances made in artillery and siege warfare tactics. After the start of the construction of the Pamplona citadel in 1571, following the trace of Jacobo Palear Fratín, this same engineer planned under orders of Philip II an ambitious project to modernize the fortifications in Fuenterrabía. There are no plans or reports of this proposal left, nor the revision made by Tiburcio Spannocchi few years later, during the nineties. Only some documents held in the Archivo General de Simancas are deepening our understanding on his proposals. Thanks to a drawing outlined on the early seventeenth century and attributed to Matteo Neroni, cosmographer at the service of the Medici, we greatly know the project of Spannocchi, and through it, the one corresponding to Fratín. The purpose of this paper is to make a formal reconstruction of the state of the fortifications of this stronghold on the early seventeenth century.
\end{abstract}

Keywords: Fuenterrabía, fortificaciones, Jacobo Palear Fratín, ingenieros militares.

\section{Introducción}

Los métodos defensivos gozaban de supremacía sobre las armas ofensivas durante la Edad Media. Pero tras la evolución de las piezas de artillería en la segunda mitad del siglo XV, sólo un cambio radical en la concepción de la fortificación podía ofrecer a las fuerzas defensivas la garantía de sobrevivir a un largo asedio. Fue Italia la nación que jugó el papel principal en la transformación durante las últimas décadas del $\mathrm{XV}$, tomando como base el ejemplo francés.

Los arquitectos militares del Renacimiento comenzaron por transformar la antigua torre medieval en una construcción capaz de alojar piezas de artillería. Lo cierto es que la aplicación de las formas poligonales triangulares como solución a los problemas planteados por los avances técnicos de la artillería fue el origen del bastión (Tzonis y Lefaivre, 1991, p. 321). La invención del bastión fue el resultado de una evolución gradual a lo largo de varias décadas, con marcados hitos puntuales (Rocolle, 1989, p. 321). Es preciso señalar como ejemplos significativos de sus inicios la fortificación de Salses, del constructor castellano Ramiro López, o Navarrés, a cargo de Pedro de Angulo.

También en España los progresos del bastión se dieron cita durante los siglos XV y XVI, 
construyéndose fortificaciones relevantes que influyeron entre los tratadistas europeos. Se produjeron grandes avances en este campo gracias al estado permanente de guerras durante la Edad Media, y la influencia clásica de griegos, fenicios, romanos, visigodos, bizantinos y árabes. Además, la temprana introducción de la artillería forzó a un mayor espesor en muros y parapetos. Esto llevó a construir torres bajas y macizas capaces de resistir y alojar la naciente artillería, e introducir tiros de flanco cruzado. En la mayoría de los casos se transformaron las fortalezas existentes adaptándolas a las nuevas exigencias defensivas. Junto con estas transformaciones también se ejecutaron nuevas fortificaciones fronterizas, en las que se hicieron planteamientos innovadores.
Hasta bien entrado el siglo XVI convivieron fortalezas medievales, renacentistas $y$ reformadas. Sin embargo, durante el resto del siglo XVI, la fortificación española no se desarrolló en la Península Ibérica al ritmo de este primer impulso debido a los constantes conflictos y enormes esfuerzos que hubo de mantener la Corona en los diversos dominios europeos y americanos. En estos desarrolló una ingente labor de obras de fortificación. De fronteras adentro, sin embargo, no pudo efectuarse una renovación de la fortificación acorde con las exigencias defensivas modernas (Quatrefages, 1984). Las únicas zonas sensibles de defensa fueron, inicialmente, los Pirineos y después las costas, sobre todo las del Mediterráneo, al aumentar el peligro turco a partir del decenio de 1520 a 1530.

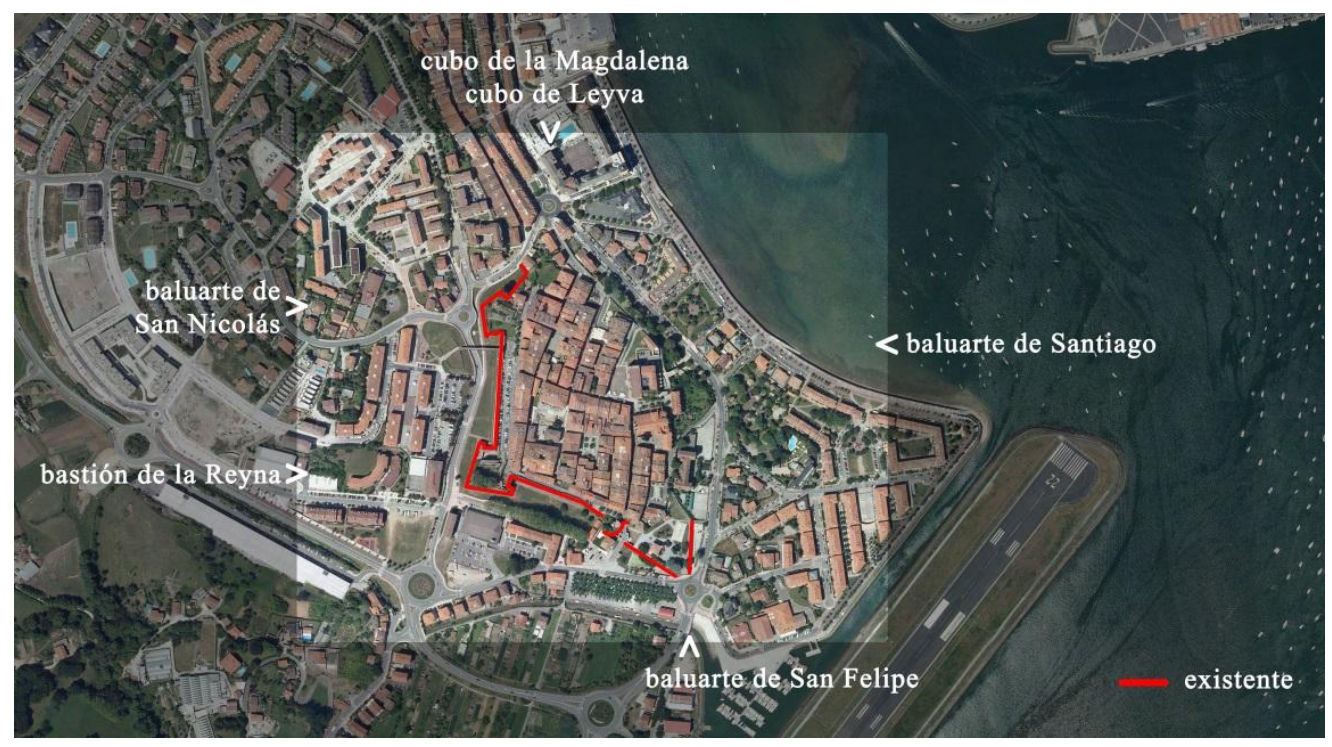

Fig. 1- Estado actual de conservación de las fortificaciones de Fuenterrabía

Tras la toma de Granada, los Reyes Católicos, conscientes de sus futuros enfrentamientos con Francia, tomaron una serie de medidas para defender la frontera septentrional de sus Estados. Se reforzaron entonces con diversas obras de fortificación, entre otras, las plazas de San Sebastián, Fuenterrabía y Pamplona. Bajo el reinado de Carlos V siguieron reforzándose estas fortificaciones fronterizas, además de realizarse extraordinarias obras de fortificación en ciudades costeras como Mallorca, Cádiz, Gibraltar, Málaga y La Coruña.

Tras la llegada de Felipe II al poder se produjeron grandes cambios en el ámbito de la fortificación (Cámara, 1989), entre los que cabe reseñar el esfuerzo del monarca por potenciar la formación técnica y científica de sus súbditos ingenieros creando la Academia de Matemáticas de Madrid. Así surgieron ingenieros expertos y tratadistas como Rojas, González de Medina 
Barba y Lechuga, y como realización la importante tarea de fortificación llevada a cabo en América (Cobos y Castro, 2005). Pero Felipe II, además de confiar en ingenieros españoles para sus territorios extra-peninsulares, importó de sus dominios de Italia ingenieros militares para las fortificaciones de la Corona, como los Fratín (Viganò, 2004), Tiburcio Spannocchi (Cámara, 1988), Juan Bautista Antonelli en las fortificaciones de la Península -entre ellas Fuenterrabía- y las posesiones de Ultramar, y Francesco de Marchi y Francesco Paciotto como diseñadores de las diversas fortificaciones construidas en Italia y Flandes (Bragard, 2011), como las ciudadelas de Amberes y Turín.

\section{Las fortificaciones renacentistas de Fuenterrabía}

$\mathrm{Al}$ igual que sucediera con núcleos de población situados en lugares estratégicos, Fuenterrabía contó desde su primitiva fundación con unas murallas medievales dominadas desde su interior por una torre defensiva. Su proximidad a Irún y Hendaya, en la desembocadura del Bidasoa, condicionó en gran medida su desarrollo social y económico. Pero fue tras la anexión de Navarra a Castilla, en el momento histórico y cultural del nacimiento de los estados modernos, cuando su ubicación de frontera natural con Francia le llevó a un auge de construcciones defensivas. Los Reyes Católicos construyeron sobre la antigua torre defensiva un castillo, que Carlos V mandó ampliar y restaurar posteriormente (Astiazaráin, 2004). Por lo que respecta al recinto medieval, al igual que sucediera en la práctica totalidad de las poblaciones, se acometieron importantes transformaciones derivadas de la necesidad de contrarrestar el efecto de las nuevas bocas de fuego y de las minas. En 1476 y 1477, sin ir más lejos, Fuenterrabía había sufrido sendos sitios a cargo de las tropas francesas durante las campañas de defensa de los derechos de Isabel la Católica frente a la Beltraneja (Pérez del Pulgar, 1476). A partir de la segunda década del siglo XVI se rebajaron torres, lienzos y almenas para disponer grandes masas de tierra ataluzada, contenidas con muros de mampostería acabados con cuidados sillares. Comenzaron a construirse los primeros bastiones. Ciertamente en Fuenterrabía se había iniciado un baluarte en 1496, un año antes del paradigmático proyecto del maestro Ramiro López para Salsas. Pero lo cierto es que, al igual que sucediera en plazas como Pamplona, muy relacionada en toda su historia con las fortificaciones que nos ocupan, la verdadera transformación fue posterior. Ni siquiera en esos momentos había evolucionado suficientemente el diseño de los bastiones modernos.

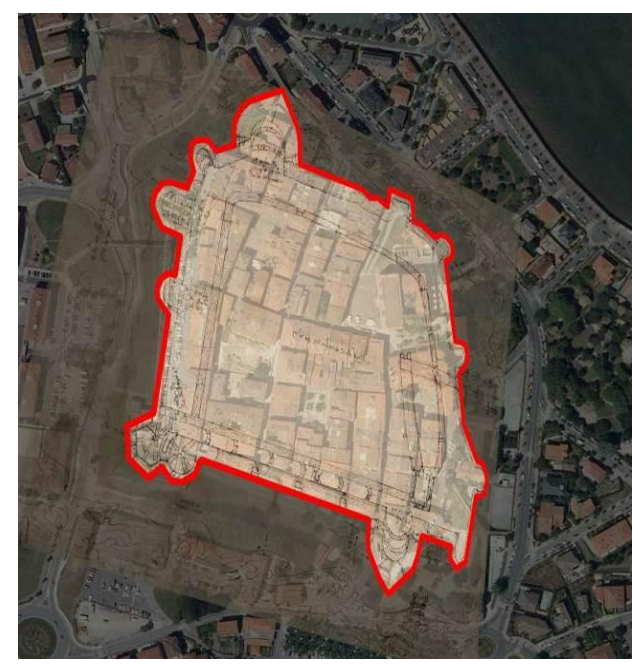

Fig. 2- Superposición de un plano de 1530 sobre la ciudad actual. Archivo General Simancas. M. P. y D. XIII-55

Fue como decíamos a partir de la segunda década de la centuria cuando se procedió a realizar un proyecto de modernización en toda regla. La forma de hacerlo fue diferente a otros casos. Debido a los condicionantes del terreno, se decidió construir un cinturón defensivo a la moderna envolviendo al medieval existente, es decir, sin modificar las antiguas torres medievales eliminándolas o sustituyéndolas en algunas casos por bastiones. El resultado fue satisfactorio en relación con la importancia estratégica territorial, en que San Sebastián y principalmente Pamplona le adelantaban (Echarri, 2000, p. 90). Así se pude apreciar en el primer documento gráfico de las fortificaciones, de hacia 1530, conservado en el Archivo General de Simancas (Fig. 2). 
En las Cortes castellanas de 1532 se propondrá acometer sin falta la finalización de las obras de fortificación comenzadas años atrás. En Fuenterrabía, al igual que sucediera en Pamplona con en el baluarte de San Llorente o San Lorenzo, se estaban levantando dos baluartes en forma de corazón, el cubo Imperial y el cubo de Leyva. Tuvieron su origen tras el asalto francés de 1521 (Astiazaráin, 2004).

El resto de baluartes que se fueron construyendo fueron significativamente pequeños, al igual que sucediera en una plaza de la importancia de Pamplona (Echarri, 2000, pp. 92-108). Se hicieron durante esos años dos: el de la Reina, que abrazaba a un cubo semicircular del recinto medieval, y otro pentagonal en la muralla nueva. Según Astiazaráin fueron obra de Pedro de Guevara y Benedito de Rávena, que habían sustituido al prestigioso ingeniero Gabriel Ladino di Martinengo, prior de Barletta (Astiazaráin, 2004). Pocos años después, al avanzar significativamente el poder destructor de la artillería, se acometieron otros bastiones de mayor escala y dotación artillera, como es el caso del de la Magdalena o San Nicolás nuevo. Nos encontramos así con que en 1521, año en que Fuenterrabía fue tomada por las tropas francesas, coexistían dos cinturones amurallados: uno medieval por la parte norte y este, y otro con capacidad artillera por los frentes sur y oeste (Fernández Antuña, 2003, p. 149).

En 1539 Carlos V visitó las fortificaciones de Fuenterrabía, dañadas en gran medida tras la recuperación de la plaza por las tropas castellanas en 1524, y como consecuencia envió poco después al capitán Luis Pizaño para que supervisara las obras de dicha plaza y las de San Sebastián. Posteriormente haría lo propio con Pamplona. Su indicación principal fue elevar el baluarte de la Reina, obra que acometería el maestro de obras Domingo de Eztala en 1545. Fue entonces cuando se acometieron las obras de fortificación abaluartada más destacadas: el baluarte de San Nicolás (1524-1545) y el baluarte de la Reina (1538-1556). Atribuidos por algunos autores al Prior de Barleta (Fernández Antuña, 2003, p. 170). Se produjo así una modernización parcial de la plaza.

\section{El proyecto de Jacobo Palear Fratín}

Durante el reinado de Felipe II se llevaron a cabo algunas obras de fortificación que asombrarían al mundo (Porreño, 1639), como la ciudadela de Amberes. En 1571 ordenó al prestigioso ingeniero militar Jacobo Palear Fratín (Viganò, 2004) que diseñara una ciudadela pentagonal del mismo estilo en Pamplona. El desarrollo de esta imponente fortaleza, junto con el resto del recinto fortificado, hizo disminuir la importancia estratégica de Fuenterrabía. El Fratín, junto con el virrey Vespasiano Gonzaga -experto poliorceta- visitó Fuenterrabía poco después, hacia 1572, y decidió, además de numerosas reparaciones en el recinto, realizar un proyecto de fortificación ambicioso. No ha quedado documentación alguna de la memoria y planos que debió elaborar. Únicamente tenemos referencias de posteriores ingenieros y de correspondencia entre el Capitán General de Guipúzcoa y el rey.

Reconstruir la propuesta del Fratín es por tanto tarea compleja. Sin embargo, contamos con la ayuda de dos planos. El primero se atribuye a Matteo Neroni (Fig. 3), cosmógrafo italiano al servicio de Felipe II (Lamberini, 2013), y se conserva en el Istituto Storico e di Cultura dell'Arma del Genio, en Roma. Recoge sin duda propuestas de la epoca, principalmente del ingeniero Tiburzio Spannocchi, de manera que algunos le han atribuido la autoría (Magiorotti, 1939). El segundo es de Spannocchi (Fig. 4), y nos referiremos a él posteriormente.

Teniendo en cuenta que la obra más destacada que propuso el Fratín fue la construcción de un nuevo baluarte más capaz en la parte sur, el baluarte de San Felipe, y sabiendo que se siguió al pie de la letra su propuesta, podemos aventurarnos a tan ardua tarea. En primer lugar tenemos la traza que hizo para el conjunto de Pamplona (Echarri, 2000), y conocemos un rasgo importante que guiaba todos sus proyectos: las contraescarpas no eran paralelas a los frentes de los baluartes, sino que los foso se ensanchaban hacia los vértices de los baluartes (Echarri, 2000, p. 146). Es una disposición insólita, contraria a lo que hacían todos los 
tratadistas. Incluso el prestigioso Francesco di Marchi los hacía divergentes pero en la dirección contraria.

Con estas premisas y teniendo en cuenta que las condiciones orográficas del lugar, a diferencia por ejemplo de Pamplona, dificultaban una ampliación del recinto, podemos lanzar la hipótesis de su proyecto según se expone en la Fig. 3.

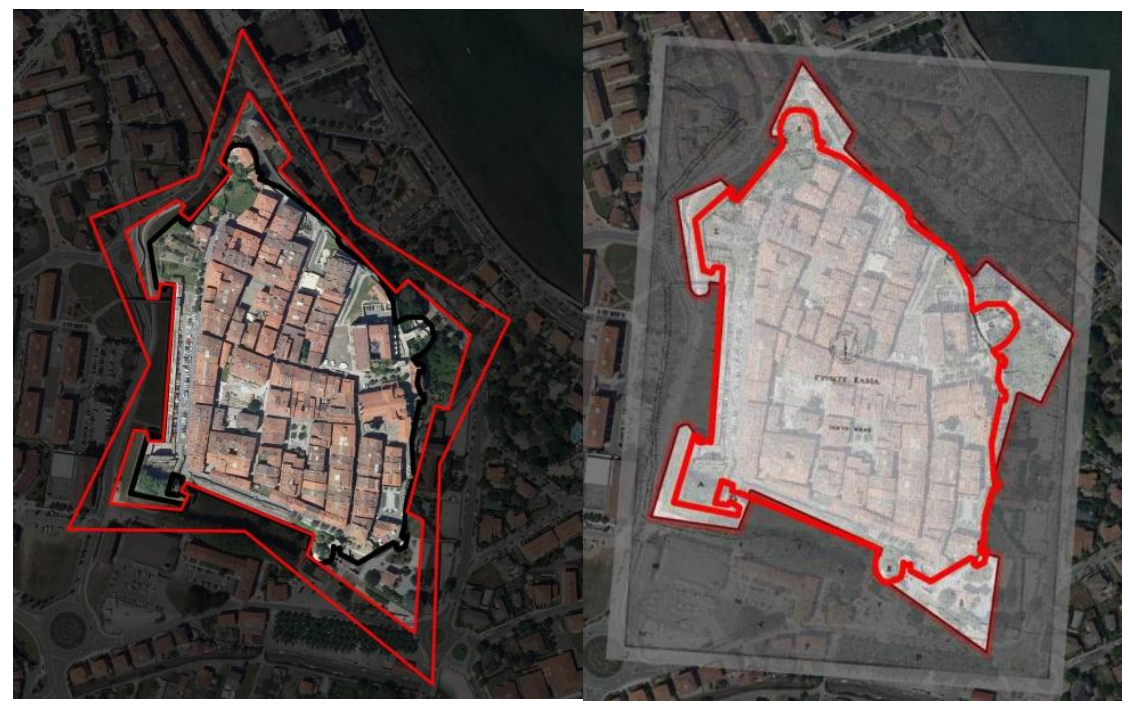

Fig. 3- Proyecto del Fratín según hipótesis de los autores. Sólo llegaría a realizarse el Baluarte de San Felipe. Plano del atlas atribuido a Mateo Neroni superpuesto sobre la ciudad actual.

Era por otra parte evidente que la proporción entre longitud de la cortina y cara del bastión iba en contra de las máximas de fortificación del momento (Cobos, 2005, p. 466). Por eso el Fratín aumentaba el tamaño de los bastiones de San Nicolás y la Reina, de forma similar al nuevo bastión de San Felipe. La distancia de flanqueo era similar a la que adoptaba el Fratín en otras plazas, es decir, en torno a 180 metros, que era una distancia adecuada para el tiro eficiente del mosquete. Por lo que respecta a las obras exteriores, principalmente revellines y hornabeques, hay que decir que, a pesar de que en esos momentos era una línea de debate entre tratadistas, todavía no se había impuesto como algo absolutamente necesario para la defensa de una plaza.

De hecho el Fratín no había introducido ninguna de estas obras en el proyecto de Pamplona. Por tanto, lo más probable es que no las introdujera en su proyecto para Fuenterrabía. Justifica esta apreciación el hecho de que tampoco Tiburcio
Spannocchi, en el proyecto que realizaría con posterioridad, apostaba por disponer revellines.

Ninguna noticia destacable nos ha llegado sobre el papel que jugó en el proyecto del Fratín el virrey Vespasiano Gonzaga. En Pamplona las disputas con el ingeniero llegaron a la Corte y las acusaciones fueron gravísimas. Ambos tenían criterios diferentes sobre el lugar en que ubicar la ciudadela. En el caso de Fuenterrabía hemos de suponer que no existieron, ya que de otra forma hubieran aparecido en los expedientes del conflicto en Pamplona (Echarri y Galiano, 2014).

\section{El proyecto de Tiburcio Spannocchi}

Poco después, en 1580, llegó a España el prestigioso ingeniero Tiburcio Spannocchi (Cámara, 1988). Su primer destino fue Fuenterrabía. Previamente había realizado un importante atlas sobre las marinas del Reino de Sicilia (BNM. Ms. $\mathrm{N}^{\circ} 788$ ), algo propio de la época y que se prolongaría a lo largo del siglo 
XVII (Orgeix, 1999; Warmoes, Orgeix y Van Den Heuvel, 2003). Una vez reconocida la plaza Spannocchi realizó un nuevo proyecto hacia 1580 (Maggiorotti, 1939, p. 168). Volvería en 1597, cinco años después de que realizara el proyecto de la ciudadela de Jaca, y en 1603, una vez nombrado Ingeniero Mayor de los Reinos de España, redactaría un informe sobre el estado de las fortificaciones, que sirvió de base para que en 1609, fallecido Spannocchi, se hiciera un nuevo proyecto de mejora en el que intervino el ingeniero Jerónimo de Soto.

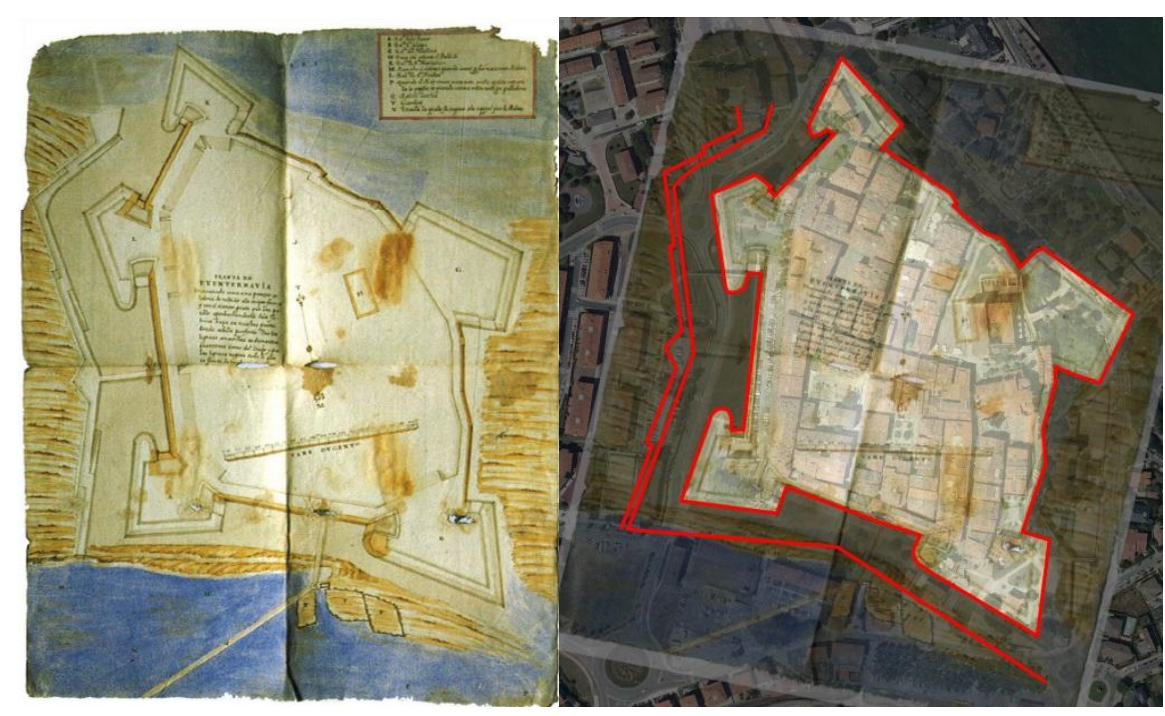

Fig. 4- Proyecto de Spannocchi en 1580. Archivo Provincial de Zaragoza. Archivo Ducal de Hijar. Fondo Idiaquez, Sala IV, leg. 199. Superposición sobre la ciudad actual.

El proyecto de Spannocchi ha quedado descrito de su propia mano a través de un informe y un preciso plano (Fig. 4) que se conservan en el Archivo Provincial de Zaragoza (Fernández Antuña, 2000). Es más que probable que coincidiera con el Fratín en lo esencial de su análisis sobre la plaza, sus ataques más desfavorables, etc. Algo similar sucede por ejemplo en sus intervenciones en Pamplona tras la muerta del Fratín, acaecida hacia finales de 1585 (Echarri, 2000, p. 153). Lo cierto es que continuó llevando a cabo las obras del baluarte de San Felipe según la traza del Fratín, que era sin duda imprescindible para mantener la defensa durante tiempo suficiente para poder recibir el socorro. Así se puso de manifiesto varias décadas después en el sitio de 1638 (Palafox, 1639; Moret, 1655). Además proponía aumentar los baluartes de la Reina y San Nicolás, de manera que pudieran alojar un mayor número de piezas de artillería, y resolver mejor el flanqueo del foso desde las casamatas.
Se ajustaban a las máximas de fortificación de la época. Completaba su proyecto con dos baluartes más en la parte que miraba a Francia. La construcción de estos dos baluartes era más compleja técnicamente. La pleamar llegaría, como sucedía en el recinto antiguo, hasta parte de su lienzo, dificultando las tareas de cimentación y consolidación, algo similar a lo que sucedía en el de San Felipe. Spannocchi creía imprescindible el baluarte que daba al arenal, ya que en bajamar quedaría la muralla casi con tierra firme por la parte de Francia, y se "hallava sin traveses".

$\mathrm{Si}$ atendemos al análisis de los contornos efectuado por nuestro ingeniero, la zona más favorable a atacante era la "de poniente de donde con comodidad se puede vatir de una colina que sobrepuja a la muralla en altura y por ser la tierra toda arvolada y con commodidad de tiera y con valles donde puede alojarse cualquier grueso exército cobierto. Por esta parte digo que los cavalleros son muy pequeños con poco través y 
sobrepujados de la sobredicha colina muy cerca a la muralla..." (AGS. G.A. leg. 110, fol. 23). Resultaba pues muy complejo proteger este frente. Posteriormente, a lo largo de los siglos XVII y XVIII se harán numerosos intentos para evitar el dominio del atacante en esta zona, aunque todos ellos sujetos a un desproporcionado coste de las obras. La más económica, aunque dudosa en su eficacia, era la que propondrían en 1636 los ingenieros militares Pedro Texeira, Antonio Gandolfo y Gerónimo Soto (Biblioteca Foral de Vizcaya, VMSS-249, en Pereda y Marías, 2004). Consistía simplemente en disponer unos caballeros con suficiente altura y capacidad para complicar los ataques y batir la colina de Santa Engracia. Teniendo en cuenta la superioridad habitual del ejército asediante -una guarnición diez veces mayor y un tren de artillería superior- esta disposición no garantizaba a priori un suficiente retraso en los trabajos del sitiador.

Terminaba su informe resaltando la necesidad de finalizar cuanto antes el baluarte de San Felipe, ya que en el estado en que se encontraba era perjudicial para la defensa.

\section{Conclusiones}

Las fortificaciones de Fuenterrabía fueron objeto de numerosos proyectos a cargo de ingenieros militares a lo largo del siglo XVI. En ellas se fueron plasmando todos los avances técnicos en la búsqueda de la máquina perfecta de defensa, que sería finalmente el bastión, aplicado en los dominios de Felipe II en fortalezas paradigmáticas como la ciudadela de Amberes.

A finales de siglo primero el Fratín y luego Spannocchi, dos de los máximos expertos que intervinieron en la Península, redactaron sendos proyectos. Ambos coincidían en la necesidad de levantar cuanto antes el bastión de San Felipe, de mayor escala que los precedentes. Diferían, según hipótesis de los autores, en el modo de disponer la contraescarpa de los fosos, un rasgo que aparecía en todos los proyectos del Fratín. Un plano atribuido a Matteo Neroni, conservado en el ISCAG de Roma, y que recoge las propuestas de Spannocchi, ayuda a recomponer cómo podría haber sido el proyecto del Fratín, así como el plano del proyecto de Spannocchi conservado en el Archivo Provincial de Zaragoza.

El informe elaborado por Spannocchi en 1580 es el primero que señala las dificultades de defender Fuenterrabía desde el interior por el poniente. Sugería la posibilidad de ampliar el recinto, aunque no era partidario por los costes que conllevaría. Era sin duda más efectivo reforzar la plaza de Pamplona, que esos momentos acometía la construcción de la ciudadela pentagonal según proyecto del Fratín.

\section{Notas}

Durante el reinado de Felipe IV, hasta el año 1638 en que los franceses sitiaron la plaza, no se llevaron a cabo obras reseñables. Fue a raíz de dicho sitio, en que los defensores consiguieron resistir hasta que llegara el socorro del Almirante de Castilla, Juan Alonso Henríquez de Cabrera, cuando se acometieron por fin algunas obras exteriores, como un revellín en frente de la puerta de San Nicolás, y una tenaza en la parte noreste. También se llevaría a cabo un fortalecimiento del frente que miraba hacia Francia, con el baluarte de Santiago y un baluarte delante del cubo de la Magdalena. Sería el comienzo de una nueva modernización del recinto que se desarrollaría a lo largo del siglo XVII.

\section{Referencias}

Astiazaráin, M. I. (2004). "El Patrimonio Militar de Fuenterrabía: el Castillo de Carlos V y las Murallas", in Orella Unzué, J. L.Historia de Fuenterrabía. Fuenterrabía, Hondarribiko Udala, pp. 477-551.

Bragard, P. (2011). Dictionnaire biographique des ingénieurs des fortifications: Pays-Bas espagnols, principauté de Liège, Franche-Comté, 1504-1713, Namur : Amis de la Citadelle de Namur.

Cámara Muñoz, A. (1988). "Tiburzio Spannocchi, Ingeniero Mayor de los reinos de España", in Espacio, Tiempo y Forma, n. 2, pp. 77-90. 
Cámara Muñoz, A. (1989). "La fortificación de la monarquía de Felipe II", in Espacio, Tiempo y Forma, n. 2, pp. 73-80.

Cobos Guerra, F. (2005). "La formulación de los principios de la fortificación abaluartada en el siglo XVI", in Técnica e Ingeniería en España: El Renacimiento, vol. I, Silva Suárez, M. (ed.), Zaragoza, Prensas Universitarias de Zaragoza, pp. 449-486.

Cobos, F.; Castro, J. (2005). "Los ingenieros, las experiencias y los escenarios de la arquitectura militar española en el siglo XVII", in Cámara, A. (coord.), Los ingenieros militares de la Monarquía Hispánica en los siglos XVII y XVIII, Madrid, Ministerio de Defensa, pp. 71-95.

Echarri Iribarren, V. (2000). Las Murallas y la Ciudadela de Pamplona. Pamplona, Departamento de Educación y Cultura-Institución Príncipe de Viana, Gobierno de Navarra.

Echarri, V.; Galiano, A. (2014). "The controversies between Jacobo Fratín and Vespasian Gonzaga on the project of the citadel of Pamplona in the late sixteenth century", in WIT Transactions on the Built Environment (Online), vol. 143, pp. 65-76.

Fernández Antuña, C.M. (2000). "Primer Informe de Tiburcio Spanochi sobre Fuenterrabía", in Boletín de Estudios del Bidasoa, $\mathrm{n}^{\circ}$ 20, pp. 67-88.

Fernández Antuña, C.M. (2003). Murallas de Hondarribia. De la cerca medieval al recinto abaluartado. Hondarribia, Antza.

Lamberini, D. (2013). Il mondo di Matteo Neroni, cosmografo mediceo, Edifir, Firenze, p. 215.

Maggiorotti, L. A. (1939). L'Opera del genio italiano all'estero. Gli architetti militari. Vol. III. Gli architetti militari italiani nella Spagna, nel Portogallo..., La Libreria dello Stato, Rome.

Moret, J. (1655). Empeños del valor, y bizarros desempeños, o Sitio de Fuente-Rabia. Translated from latín 'De obsidione Fontirabiae: libri tres' by Silvestre de Arlegui, M. \& J. M.Ezquerro, in Pamplona 1763. Tolosa, Imprenta, librería y encuadernación de Eusebio López, 1893.

Orgeix d', É. (1999). "Aperçu d'un genre iconographique peu connu: les atlas militaires manuscrits de la première moitié du XVIIe siècle", in Le paysage des cartes, genèse d'une codification. BousquetBressolier, C. París, Musée des Plans Reliefs, pp. 29-48.

Palafox y Mendoza, J. (1639). Sitio y socoro de Fuenterabia y sucesos del año de mil y seiscientos y treinta y ocho. 4th print, 1793, Madrid, Don Gerónimo Ortega y herederos de Ibarra.

Pereda, F.; Marías, F. (2004). "De la cartografía a la corografía: Pedro Texeira en la España del Seiscientos", in Ería, vols. 64-65, pp. 129-157.

Pérez del Pulgar, F. (1476). Crónica de los Reyes Católicos. Cap. LVI. El ataque francés a Fuenterrabía.

Porreño, B. (1639). Dichos y hechos del Señor Rey Felipe Segundo, el Prudente, Potentísimo y Glorioso Monarca de las Españas y de las Indias. Sevilla, Pedro Gómez Pastrana, cap. XII.

Quatrefages, R. (1984). "La fortificación en España durante el Renacimiento (II)”, In Ejército, february 1984, p. 74.

Rocolle, P. (1989). 2000 ans de fortification française. Vol. 2, Du 16e siècle au mur de l'Atlantique. Lavauzelle. Paris, p. 321.

Tzonis, A., Lefaivre, L. 1991. El bastión como mentalidad. In La ciudad y las murallas. Seta, C. De and Le Goff, J. (eds.). Ed. Cátedra. Madrid, p. 321.

Viganò, M. (2004). «El fratin mi ynginiero». I Paleari Fratino da Morcote ingegneri militari ticinesi in Spagna (XVI-XVII secolo), Bellinzona, Edizioni Casagrande.

Warmoes, I., Orgeix d', É. and Van Den Heuvel, C. (2003). Atlas militaires manuscrits Européens (XVIe-XVIIIe siècles). París, Musée des Plans Reliefs. 